\title{
Sound propagation in liquid He in impurity-helium solids
}

\author{
S. I. Kiselev, V. V. Khmelenko, and D. M. Lee \\ Laboratory of Atomic and Solid State Physics, Cornell University, Ithaca, NY 14853-2501, USA \\ E-mail: khmel@ccmr.cornell.edu
}

Received April 6, 2000

\begin{abstract}
The observed features of attenuation of ultrasound in $\mathrm{Im}-\mathrm{He}$ samples created after introduction of impurity particles $\left(D_{2}, N_{2}, \mathrm{Ne}, \mathrm{Kr}\right)$ in a volume of helium II show that a porous substance consisting of a loosely interconnected continuous network is created. It is formed by impurity particles encapsulated in solidified helium. The propagation of ordinary sound in these porous samples is similar to the fast sound mode in light aerogels. The temperature dependence of attenuation for different $\mathrm{Im}-\mathrm{He}$ samples is investigated. It is established that the character of attenuation in $\mathrm{D}_{2}-\mathrm{He}$ samples is considerably different from that in heavier $\mathrm{Im}-\mathrm{He}$ solids $\left(\mathrm{Im}=\mathrm{N}_{2}, \mathrm{Ne}, \mathrm{Kr}\right)$. Analysis of attenuation lets us conclude that $\mathrm{Im}-\mathrm{He}$ samples have a wide distribution of pores from $8 \mathrm{~nm}$ to $800 \mathrm{~nm}$. The study of ultrasound in helium in $\mathrm{Im}-\mathrm{He}$ samples near the $\lambda$-point shows the presence of broadening in the attenuation peak as compared with bulk liquid helium. The suppression of $T_{c}$ is very small, $\leq 0.2 \mathrm{mK}$.
\end{abstract}

PACS: $67.40 . \mathrm{Yv}, 67.40 . \mathrm{Mj}$

\section{Introduction}

The investigation of neutral atoms and clusters in liquid and solid helium is a rapidly developing research field [1-4]. Much progress has been achieved in studies of the spectral characteristics of single atoms or molecules trapped in matrices of solid helium or dissolved in liquid helium, from which information about the structure of the helium surrounding these impurities was found.

The impurities can be divided into two classes according to the sign of chemical potential inside the helium matrix. Particles with a positive potential tend to form bubbles, while atoms with a negative potential create snowballs. In the latter case, after introducing the impurity particles into liquid helium, we can produce stable impurity-helium ( $\mathrm{Im}-\mathrm{He})$ clusters, which make it possible to create macroscopic Im-He samples consisting of impurity atoms isolated in liquid or solid helium. At first these systems were obtained by injecting atoms and molecules such as nitrogen, deuterium, neon and krypton [5-7] into superfluid helium. These metastable systems are of fundamental interest. For example, there is the possibility of observing collective effects caused by the interaction of stabilized impurity particles in helium and also the opportu- nity to create new materials with high energy density stored in them [8]. A very high relative concentration of nitrogen atoms in the solidified helium $(\mathrm{N}: \mathrm{He}=4 \%)$ has already been achieved by injecting the products of a nitrogen-helium discharge into a volume of superfluid helium [6,9]. For this case, the density of the chemical energy stored in these samples $\left(\sim 5 \cdot 10^{3} \mathrm{~J} / \mathrm{gm}\right)$ is close to that of the best chemical explosive materials. Another interesting aspect of Im-He systems is the possibility of chemical reactions in a solid matrix when the state of the low-temperature matrix is mostly determined by zero-point motion [10-12]. Investigations of atoms and molecules of hydrogen isotopes stabilized in superfluid helium have revealed tunneling reactions resulting in the exchange of hydrogen and deuterium atoms between the atomic state and the bound molecular state [5].

Investigations of macroscopic solid samples formed by injecting impurities into superfluid helium have opened the possibility for the creation of metastable solid phases built from coalescing clusters of solid helium surrounding the impurity particles. Later it was shown that the centers of the these clusters might consist of either single impurity particles or small clusters of impurities [13,14]. At the same time the structure of these $\mathrm{Im}-\mathrm{He}$ solids is 
not fully determined. Recently x-ray spectroscopy showed that the impurities (surrounded by a few layers of solid helium) formed porous structures in superfluid helium [15]. The characteristic size of the constituent building blocks of this porous material is $6 \mathrm{~nm}$. The density of impurity particles can be as high as $10^{20}$ atoms $/ \mathrm{cm}^{3}$ (with a volume fraction $\sim 0.5 \%$ ).

We briefly summarize the present state of knowledge regarding the $\mathrm{Im}-\mathrm{He}$ solids. The preponderance of evidence suggests that macroscopic samples of the Im-He solid phase are built from aggregations of small Im-He clusters. Furthermore, we believe that these aggregates form extremely porous solids into which liquid helium can easily penetrate. They consist of a loosely connected continuous network of impurities or clusters of impurities each of which is surrounded by one or two layers of solidified helium. Therefore we have a unique opportunity to investigate the properties of superfluid helium in porous structures formed by particles with a well known potential of interaction with helium.

A great deal of effort has recently been dedicated to the investigation of superfluid helium in porous materials. We cite here a recent review article describing the specific features of helium in various porous structures [16]. The importance of these studies is now discussed. Superfluidity of helium in restricted geometries has been the object of much theoretical and experimental interest in recent years. Helium has long provided a testing ground for theories of phase transitions. Bulk helium exhibits three-dimensional $(3 D)$ critical behavior near lambda transitions, while helium films on flat substrates are $2 D$ with a vortex-inhibiting transition of the Kosterlitz-Thouless type [17]. When helium is adsorbed in a porous medium either as a film or completely filling the pores, its behavior may be changed in a number of ways. Finite size effects might shift or even smear out the phase transition, the multiply-connected substrate geometry may change the effective dimensionality, or disorder induced by the porous material may change the nature of the transition. The superfluid density, $\rho_{s}$ vanishes near the lambda-point according to the power law:

$$
\rho_{s}(t)=\rho_{s 0}|t|^{\zeta}
$$

where $t$ is a reduced temperature $\left(t=\left(T-T_{c}\right) / T_{c}\right)$ with transition temperature $T_{c}$. The superfluid density exponent $\zeta$ is found to be 0.6705 for bulk helium [18], for helium in Vycor glass [19] and for helium in porous gold [20]. For a particular aerogel it is significantly larger -0.81 [19]. For different porosity aerogels it changes from 0.71 to 0.81 [21]. At the same time the superfluid transition temperature is suppressed down to $1.955 \mathrm{~K}$ in Vycor. For porous gold (which contains larger pores) $T_{c}=$ $=2.1691 \pm 5 \cdot 10^{-5} \mathrm{~K}$, and for different porosity aerogels the suppression is very small: $T_{c}=2.16985 \pm$ $\pm 3 \cdot 10^{-5}$ ( in $95 \%$ aerogel), $\quad T_{c}=2.1717 \pm 1 \cdot 10^{-5}$ (in $99.5 \%$ aerogel) [21]. In the light of these previous studies, the problem of investigating the critical behavior of helium near the lambda point in the new class of porous material discussed here arises quite naturally. There are experimental difficulties in combining the method of preparing impurity-helium solids with precise heat capacity or torsional oscillator techniques. On the other hand, ultrasonic velocity and attenuation measurements $[22,23]$ can be easily applied to investigate superfluid helium in $\mathrm{Im}-\mathrm{He}$ solids. The sound velocity in porous media can provide information about the superfluid density as well as the density and elastic properties of the solid matrix. The sound attenuation reflects the dissipation in the system, and its frequency dependence is related to the characteristic pore size [24]. Also, if the sound speed in a «dry» sample (a sample with liquid helium removed) could be measured, we could then determine the effective density of the Im-He solid. This turns to be extremely difficult and has not as yet been accomplished.

The motion of a fluid in a porous medium during acoustic measurements depends on the pore size and the fluid's properties. In liquid ${ }^{4} \mathrm{He}$ the viscous penetration depth is

$$
\delta_{\text {visc }}=\left(2 \eta / \omega \rho_{n}\right)^{1 / 2},
$$

where $\eta$ is the viscosity of ${ }^{4} \mathrm{He}, \rho_{n}$ is the density of the normal component and $\omega$ is the frequency of ultrasound. At low sound frequencies, $\delta_{\text {visc }}$ is bigger than the pore size so the entire normal component is viscously locked to the solid matrix. Therefore the main effect of the fluid is to change the effective density of the porous medium. At high frequencies, only a thin surface layer is dragged along with the solid. The effective density of the porous material then is much smaller and the sound is strongly attenuated by the viscous losses in the surface layer. To use sound for probing the structure of porous material, one should vary $\delta_{\text {visc }}$ over as large a range as possible. Superfluid helium gives us this unique opportunity. Between 1.0 to $2.17 \mathrm{~K}$ the normal fluid density fraction varies from zero to one, causing $\delta_{\text {visc }}$ to change by an order of magnitude from $1500 \mathrm{~nm}$ to $100 \mathrm{~nm}$ for $5 \mathrm{MHz}$ sound. Biot created a basic theoretical framework for sound propagation 
in porous materials $[25,26]$. He considered the flow of the viscous fluid under an oscillatory pressure gradient in elastic porous solids. In the low frequency regime his theory predicted that attenuation changes as [24]

$$
\alpha \propto \rho_{n}^{2} \omega^{2} / \eta
$$

For high frequency sound, the corresponding attenuation is

$$
\alpha \propto \sqrt{\eta \rho_{n} \omega} .
$$

In this paper we report results of ultrasound measurements of velocity and attenuation of longitudinal waves in helium filled porous Im-He solids. Some of the results have been published in our previous paper [27]. It was found that the speed of sound in this material is close to and decreases more rapidly with temperature than first sound in bulk liquid helium, similar to behavior observed in aerogel [28]. There was no clear explanation, however, to the way the attenuation of ultrasound changes with temperature in helium filled Im-He solids. Here we present the results of more detailed investigations of the speed and attenuation of sound, particularly near the lambda-point. One goal of this work was to check a possible $T_{c}$ suppression. We also performed the measurements at different frequencies $(1,3,5 \mathrm{MHz})$ to help us to understand the mechanism of attenuation. Moreover we investigated the stability of different Im-He samples between 1 and $4.2 \mathrm{~K}$.

\section{Experimental method}

\subsection{Preparation of porous impurity-helium solids}

The technique for creating impurity-helium solids in a volume of $\mathrm{He}$ II was similar to that developed by the Chernogolovka group [6,29]. A gas jet of helium containing a small fraction (0.5$1 \%$ ) of impurity atoms or molecules impinged onto superfluid helium contained in a small dewar beaker sitting in the main helium glass dewar. The helium vapor pressure in the dewar was maintained at 1-5 Torr by a high speed rotary pump. The gas entered through a quartz capillary of diameter about $0.7 \mathrm{~mm}$, near the end of which was a region containing a high power RF (60 MHz) discharge for dissociation of molecules. In this series of experiments most of the Im-He samples were created by introducing into He II a gas jet not subjected to the action of the RF discharge. When there was no need to dissociate molecules we used a stainless steel capillary with inner diameter of $1.6 \mathrm{~mm}$ sur- rounded by a vacuum jacket with a heater at the bottom end. As in all of our previous setups, the diameter of the hole at the end of the capillary was $0.7 \mathrm{~mm}$. The nozzle of the capillary was located $2 \mathrm{~cm}$ above the surface of the superfluid helium in the small quartz dewar mentioned above, which acted as the collection beaker. To prevent the freezing of impurities in the nozzle we heated the end of the capillary by an annular heater $(R \sim 10 \Omega)$. In order to keep the level of helium in the beaker constant, a continuously operating fountain pump was used. When the gas mixture jet impinged on the surface, a macroscopic snowflake-like semitransparent material was created. This fell down through the liquid and then congealed, forming a porous impurity-helium solid between the transducers of the ultrasound cell. The centers of transducers were $\sim 5 \mathrm{~cm}$ below the level of helium in the beaker. For more effective collection of the sample in the cell we used a quartz funnel with two side plates which were placed between the endplates of the cell. On some occasions, in order to compress the sample at low temperatures we used a small teflon cylinder which could be moved up and down. We can monitor the presence of the sample in the cell and its homogeneity visually through slits on the sides of the glass dewars. In these experiments the impurities used were $\mathrm{Kr}$, Ne and molecular $\mathrm{D}_{2}$ and/or $\mathrm{N}_{2}$. Gas mixtures of $\operatorname{Im}: \mathrm{He}=1: 100$ were used to dilute the impurity particles and therefore prevent them from congealing as they passed from the source to the surface of the liquid helium. The total flux of the gas mixtures was (4-6) $\cdot 10^{19}$ particles /s. Samples with a visible volume between 1.2 and $1.7 \mathrm{~cm}^{3}$ were usually used.

\subsection{Ultrasound cell}

In our experiments we used two different ultrasound cells. In the first cell two x-cut gold plated quartz transducers (5 $\mathrm{MHz}$ fundamental) were used [27]. The crystals were $1 \mathrm{~cm}$ in diameter. Each of these was pushed against the parallel walls of the cell by two springs, one of which served as a central electrode. The ground was provided by the brass body of the cell. The path length was determined at room temperature with a micrometer with a correction being made for the contraction upon cooling. The value for the path length used in the experiments was $(1.572 \pm 0.005) \mathrm{cm}$. In our second cell we used two $\mathrm{LiNbO}_{3}$ transducers with fundamental frequency $\sim 1 \mathrm{MHz}$ (Fig. 1). The odd harmonics were also used ( 3 and $5 \mathrm{MHz}$ ). The transducers were $1.3 \mathrm{~cm}$ in diameter. Otherwise the design of the second cell was similar to the first one except 


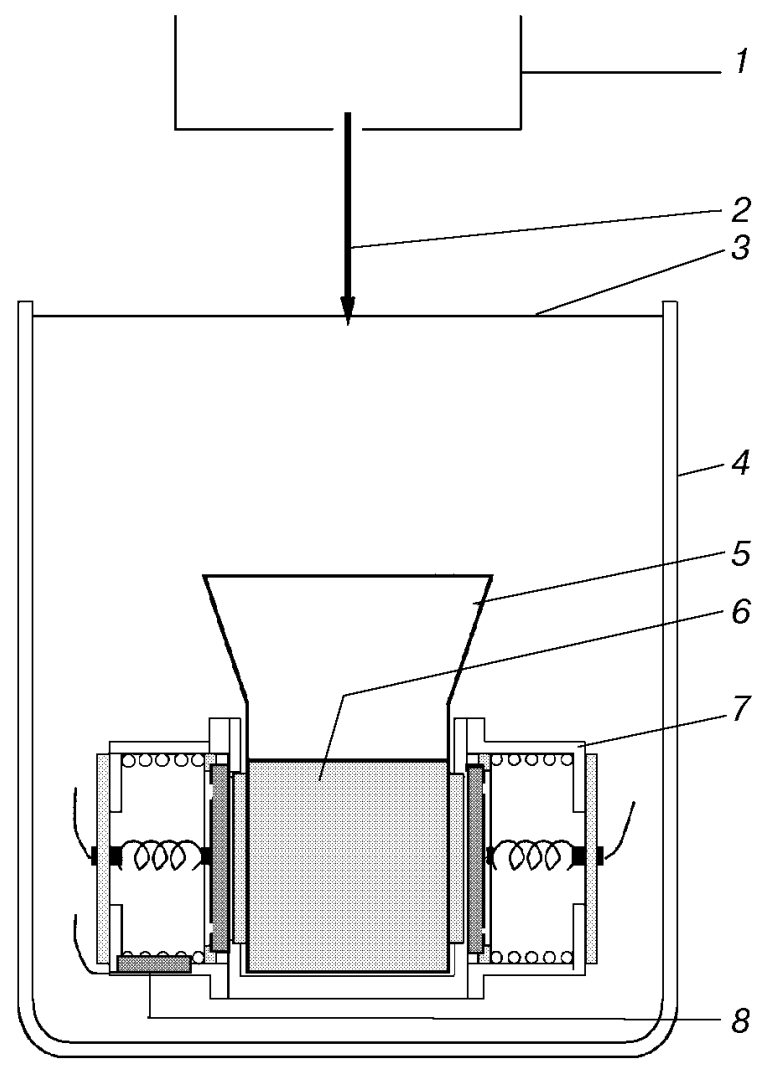

Fig. 1. Experimental cell: 1 - atomic and molecular source; 2 - impurity-helium jet; 3 - surface of liquid helium; 4 quartz dewar; 5 - quartz funnel; 6 - impurity-helium solid; 7 - ultrasound cell; 8 - germanium thermometer.

that the distance between transducers was $(1.470 \pm 0.005) \mathrm{cm}$.

\subsection{Spectrometer}

The ultrasonic measurements were made using a homodyne phase-sensitive spectrometer (Fig. 2). A continuously operating oscillator was gated to provide a transmitter pulse of $4-12 \mu \mathrm{sec}$. The amplitude of the input signal could be varied from 1 to $100 \mathrm{~V}$ at the resonant frequency or at the odd harmonics of the transmitting crystal. The ultrasonic pulse was received by the second crystal, amplified and split in two parts, one of which was used to directly measure the attenuation by recording the amplitude of the signal on a TEK460 digital oscilloscope. The second part was split again in two parts to obtain the $0^{\circ}$ and $90^{\circ}$ components, $A_{0^{\circ}}$ and $A_{90^{\circ}}$. They where used to determine the phase of the signal $\varphi$ :

$$
\operatorname{tg}(\varphi)=A_{0^{\circ}} / A_{90^{\circ}} .
$$

Once the initial speed of sound was measured at the temperature $T_{0}$ from the pulse transit time $\tau_{0}$,

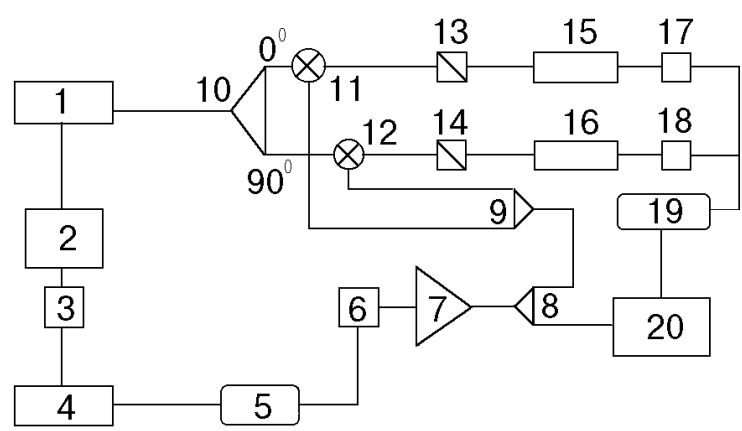

Fig. 2. Ultrasound spectrometer: 1 - HP8656B signal generator; 2 - MATEC310 gated amplifier; 3, 6 - step attenuators $(0-100 \mathrm{~dB}) ; 4$ - ENI325LA power amplifier $(+50 \mathrm{~dB})$; 5 - ultrasound cell; 7 - amplification stage $(+70 \mathrm{~dB}) ; 8,9-$ power splitters; 10 - hybrid power splitter; 11, 12 - mixers; 13, 14 - low pass filters; 15, 16 - PAR160 box car integrators; 17,18 - multimeters; 19 - computer; 20 - TEK TDS 460 oscilloscope for peak-peak and time of flight measurement.

changes in velocity were calculated from the phase of the received signal.

$$
\Delta v=l\left(\tau_{0}+\frac{\varphi(T)-\varphi\left(T_{0}\right)}{\omega}\right)^{-1},
$$

where $l$ is the length of the cell. With typical samples the changes in velocity of a few parts per million could be resolved. A second oscilloscope was used to display the signal on a longer time scale. It registered up to 12 echoes of the signal in the first cell but only 3 echoes in the second one.

\subsection{Thermometry}

For temperature measurements, a calibrated Lake Shore germanium resistor was used. The thermometer was located inside the base of the cell just outside the path of the ultrasonic pulses so that the effect of the temperature difference between the thermometer site and the sound path is minimized. After the lowest temperature (1.0-1.1 K) was achieved through pumping by both the rotary pump and a roots blower, the initial speed of sound was measured. In the next stage, by closing the pumping line down the temperature was allowed to increase by $\sim 10^{-4} \mathrm{~K} / \mathrm{s}$. Near the lambda-point the rate was decreased to about $10^{-6} \mathrm{~K} / \mathrm{s}$. During the warmup, the fountain pump was constantly supplying helium into dewar beaker up to the lambdapoint. After the superfluid transition, the warmup rate increased to $10^{-4} \mathrm{~K} / \mathrm{s}$ but boiling in the inner dewar did not occur. 

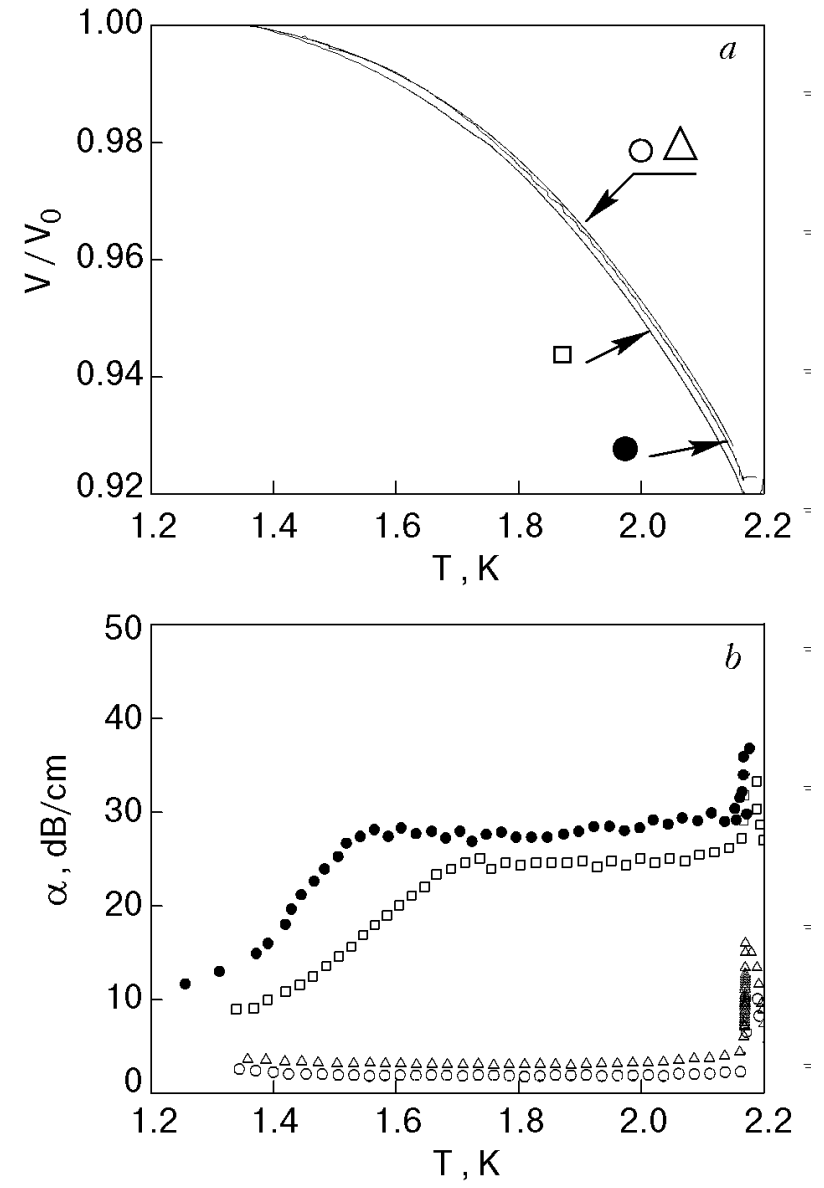

Fig. 3. The velocity (a) and attenuation (b) of $4.96 \mathrm{MHz}$ sound in liquid helium: in bulk $(O)$, in $\mathrm{D}_{2}-\mathrm{He}$ solid $(\Delta)$, in $\mathrm{Ne}-\mathrm{He}$ solid $(\square)$, in $\mathrm{Kr}-\mathrm{He}$ solid $(\mathbf{\bullet}) . V_{0}$ is the ultrasound velocity at initial temperature for these measurements, $T=1.362 \mathrm{~K}$.

\section{Experimental results}

Figure 3 shows the results of $5 \mathrm{MHz}$ ultrasound measurements (in the first cell) at $T=1.1-2.2 \mathrm{~K}$ in different $\mathrm{Im}-\mathrm{He}$ solids $\left(\mathrm{Im}=\mathrm{D}_{2}, \mathrm{Ne}, \mathrm{Kr}\right)$ just after preparation. Here and in later figures we show for comparison the velocity and attenuation of sound in bulk helium which were measured in each experiment before accumulating the sample. The attenuation of sound in the presence of $\mathrm{Im}-\mathrm{He}$ samples (for such heavy impurities as $\mathrm{Ne}, \mathrm{Kr}$ ) is larger than in bulk helium at low temperatures and increases rapidly with temperature, after which it reaches a plateau and at the $\lambda$-point it goes through a maximum. Whereas heavy Im-He samples all have similar characteristic features, the $\mathrm{D}_{2}-\mathrm{He}$ solid behaves quite differently. In the latter case, we do not observe any measurable effect on the speed of sound, and attenuation has a behavior similar to that of bulk liquid helium, although slightly $(\sim 1 \mathrm{~dB} / \mathrm{cm})$ higher. The samples produced with heavy impurities are much denser than

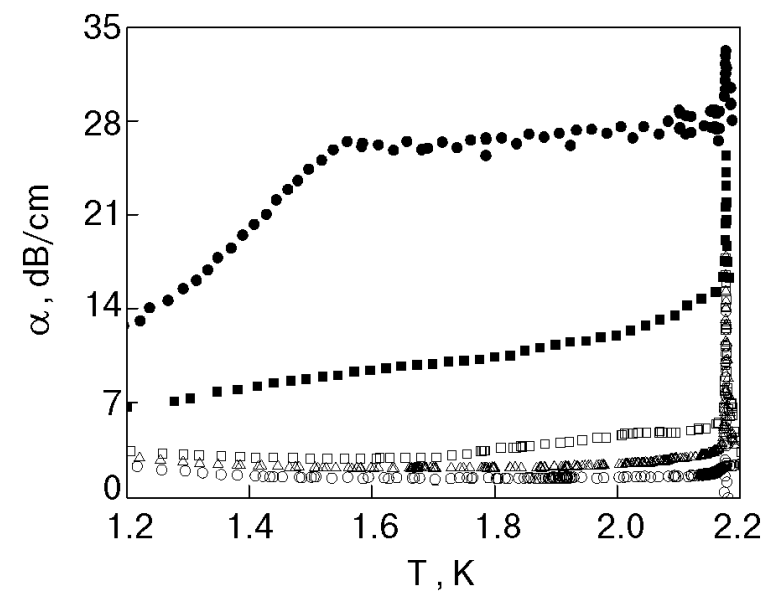

Fig. 4. The attenuation of $5 \mathrm{MHz}$ ultrasound in liquid helium: in bulk $(\mathrm{O})$, in $\mathrm{D}_{2}-\mathrm{He}$ solid $(\Delta)$, in $\mathrm{D}_{2}-\mathrm{N}_{2}-\mathrm{He}$ solid (prepared without discharge $(\square)$, with discharge $(\square)$ ), in $\mathrm{N}_{2}-\mathrm{He}$ solid (๑)

the ones with the $\mathrm{D}_{2}$ impurity. In the case of the heavy impurities, investigations become impossible above temperature in the neighborhood of $1.4 \mathrm{~K}$ because of an extremely high attenuation. We could have increased the signal by decreasing the distance between the transducers but the method of collecting the sample does not allow us to do that. Therefore, in a series of experiments we introduced pulses with very large (up to $100 \mathrm{~V}$ ) values of the input amplitude. From the analysis of attenuation in different Im-He samples it became clear that $\mathrm{D}_{2}-$ (heavy $\mathrm{Im}$ )-He samples are, in fact, the most suitable ones for investigations of mechanisms of attenuation, because they have a relatively small attenuation. In this work we compare three samples: $\mathrm{N}_{2}-\mathrm{He}, \mathrm{D}_{2}-\mathrm{He}$ and mixed $\mathrm{D}_{2}-\mathrm{N}_{2}-\mathrm{He}$. We investigated the stability of these samples and also the frequency dependence of ultrasound attenuation. Measurements were performed between $1.0 \mathrm{~K}$ and $4.2 \mathrm{~K}$, with special attention to the region near the $\lambda$-point. Figure 4 shows the characteristic temperature dependence of attenuation for these three samples. The behavior of the attenuation in the $\mathrm{D}_{2}-\mathrm{N}_{2}-\mathrm{He}$ solid repeats that of the $\mathrm{D}_{2}-\mathrm{He}$ solid and bulk helium up to $T \sim 1.75 \mathrm{~K}$. Warming up further, it becomes closer to the behavior of the $\mathrm{N}_{2}-\mathrm{He}$ solid, i.e. the attenuation increases and then reaches a plateau.

\subsection{Stability of the structure of Im-He solids}

In this section we discuss the factors affecting the structural stability of Im-He samples. Changes in the structure should lead to changes in the attenuation of ultrasound. Earlier it was discovered that the compression of samples results in an increase of attenuation [27]. In this series of experi- 


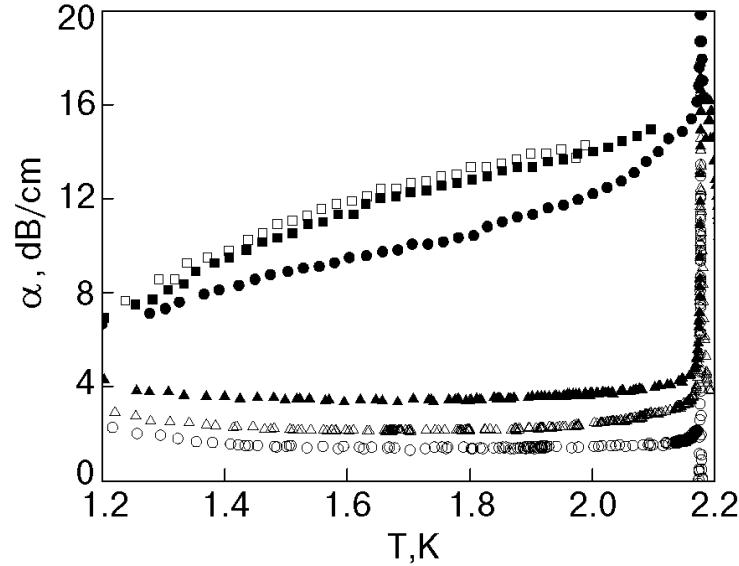

Fig. 5. The attenuation of ultrasound in liquid helium: in bulk $(O)$, in $\mathrm{D}_{2}-\mathrm{He}$ solid (after preparation of solid $(\Delta)$, after crossing $\lambda$-point and cooling down $(\mathbf{\Delta})), \mathrm{D}_{2}-\mathrm{N}_{2}-\mathrm{He}$ solid prepared with discharge (after preparation ( $)$, after crossing $\lambda$-point and cooling down ( $(\mathbf{\square})$, after warming up to $2.1 \mathrm{~K}$ and cooling down ( $\square)$ ).

ments we show the impact of warmup from $1.0 \mathrm{~K}$ to $2.2-4.2 \mathrm{~K}$ on the structure of our samples. Figure 5 presents the results of ultrasound measurements for the three samples mentioned above as they were warmed up in the temperature ranges below or above $T_{\lambda}$. We can see that as the sample was heated up to $T_{\max }<T_{\lambda}$ and then cooled again, there was no change in attenuation and therefore the structure did not change. Crossing $T_{\lambda}$ always led to the small transformations in the structure registered by a slight increase of sound attenuation. The attenuation preserved its characteristic features, nevertheless.

Figure 6 shows the attenuation of ultrasound in liquid helium filled $\mathrm{D}_{2}-\mathrm{N}_{2}-\mathrm{He}$ solid during warmup from $1 \mathrm{~K}$ to $4.2 \mathrm{~K}$. It was found that a sudden drop in attenuation takes place at $3.4-3.5 \mathrm{~K}$, which

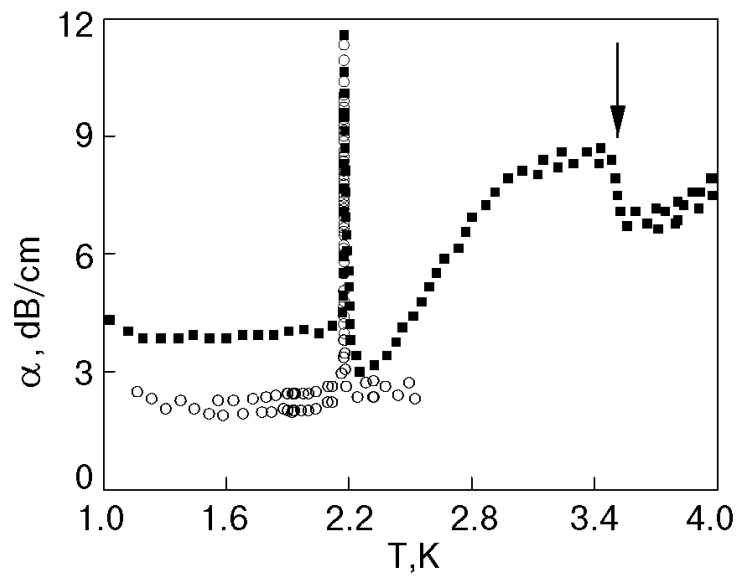

Fig. 6. The attenuation of $3.16 \mathrm{MHz}$ ultrasound in liquid helium: in bulk (O), $\mathrm{D}_{2}-\mathrm{N}_{2}-\mathrm{He}$ solid ( $\left.\boldsymbol{\square}\right)$.

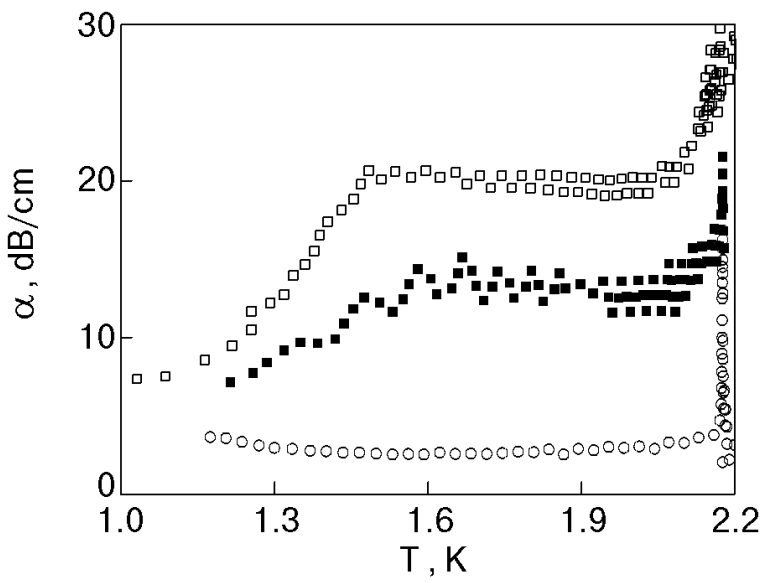

Fig. 7. The attenuation of ultrasound in liquid helium: in bulk $(\mathrm{O})$, in $\mathrm{N}_{2}-\mathrm{He}$ solid at $3.16 \mathrm{MHz}(\boldsymbol{\square})$, at $5.33 \mathrm{MHz}(\square)$.

is attributed to the change of structure of this solid. Below this temperature, the sample occupied the whole cell, but at $T \sim 3.5 \mathrm{~K}$ a significant compression of the solid by a factor of 12 was observed. This is the first observation of a spontaneous macroscopic change of structure of an Im-He sample in liquid helium. For $\mathrm{N}_{2}-\mathrm{He}$ without $\mathrm{D}_{2}$, these changes were not observed.

\subsection{Frequency dependence of the sound attenuation}

Figure 7 presents the frequency dependence of the attenuation of ultrasound in the $\mathrm{N}_{2}-\mathrm{He}$ sample. Decreasing the frequency leads to a lower attenuation and also shifts the point where the attenuation levels off to a plateau to higher temperatures. Figure 8 shows the frequency dependence of attenuation in $\mathrm{D}_{2}-\mathrm{N}_{2}-\mathrm{He}$ sample measured at 3 and $5 \mathrm{MHz}$. It significantly differs from the one observed in heavy Im-He samples (like $\mathrm{N}_{2}-\mathrm{He}$ ). For the first time, for the $\mathrm{D}_{2}-\mathrm{N}_{2}-\mathrm{He}$ solid, we detected a steady and steep

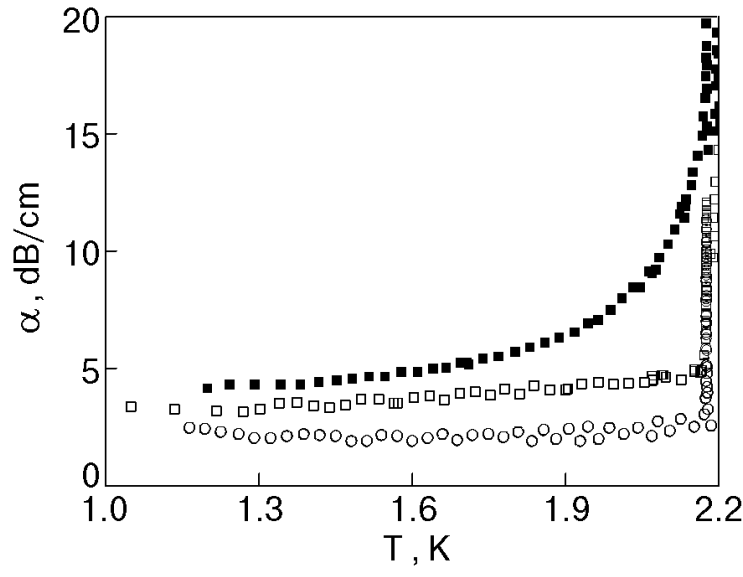

Fig. 8. The attenuation of ultrasound in liquid helium: in bulk (O), in $\mathrm{D}_{2}-\mathrm{N}_{2}-\mathrm{He}$ solid at $3.16 \mathrm{MHz}(\square)$, at $5.33 \mathrm{MHz}(\boldsymbol{\square})$. 


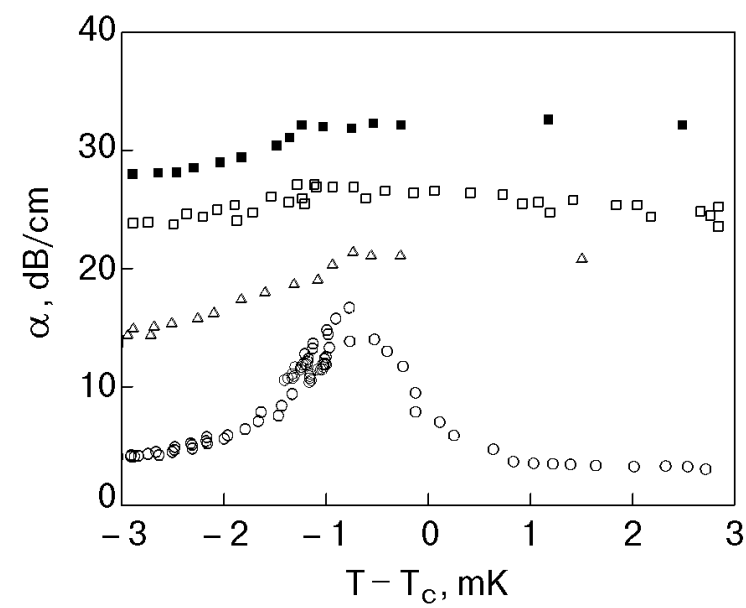

Fig. 9. The behavior of attenuation of $4.96 \mathrm{MHz}$ sound in liquid helium near $\lambda$-point: in bulk $(O)$, in different $\mathrm{N}_{2}-\mathrm{He}$ solids $(\Delta, \square, \boldsymbol{\square})$. For $\mathrm{N}_{2}-\mathrm{He}$ solids the attenuation is shifted by 0,5 and $10 \mathrm{dBm} / \mathrm{cm}$, correspondingly.

monotonic increase in the attenuation up to the $\lambda$-point without occurrence of a plateau for $5 \mathrm{MHz}$ sound. This might be explained by the purely high frequency behavior of the attenuation in this sample. For $3 \mathrm{MHz}$ attenuation we observed only a slow linear increase in the same temperature range.

\subsection{The behavior of the sound attenuation and the speed of sound near $T_{c}$.}

The results of measurements near the $\lambda$-point for $\mathrm{N}_{2}$-He solids are shown in Fig. 9. Different symbols represent different samples. The maxima of attenuation near the $\lambda$-point for $\mathrm{N}_{2}-\mathrm{He}$ samples are much broader than for liquid helium, making the precise determination of the position of the maxima impossible. We can conclude from this data that there is

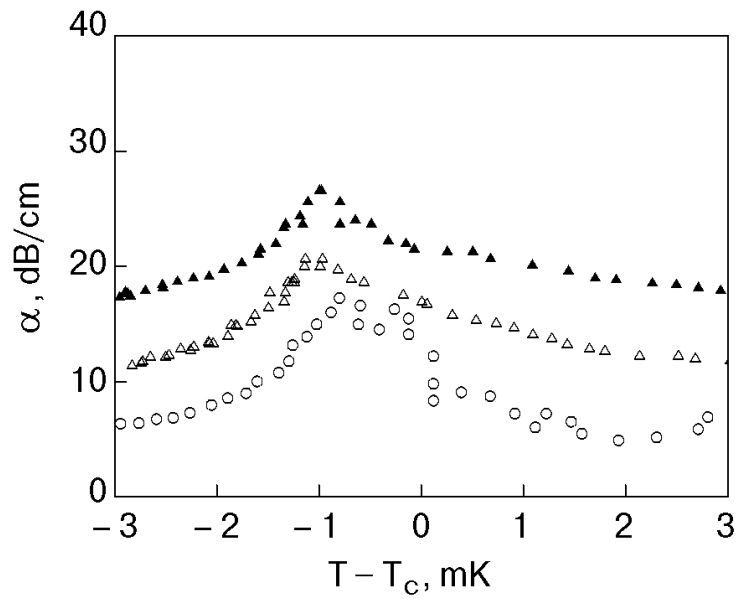

Fig. 10. The behavior of attenuation of ultrasound in liquid helium near $\lambda$-point: in bulk $(O)$, in different $\mathrm{D}_{2}-\mathrm{N}_{2}-\mathrm{He}$ solids $(\Delta, \boldsymbol{\Delta})$. For $\mathrm{D}_{2}-\mathrm{N}_{2}-\mathrm{He}$ solids the attenuation is shifted by 5 and $10 \mathrm{dBm} / \mathrm{cm}$, correspondingly. no significant shift of the $\lambda$-point for helium-filled porous $\mathrm{N}_{2}-\mathrm{He}$ solids.

Figure 10 shows the attenuation of ultrasound (only $5 \mathrm{MHz}$ data is presented) in the mixed $\mathrm{D}_{2}-\mathrm{N}_{2}-\mathrm{He}$ solids. The width of attenuation peak near the $\lambda$-point is only slightly broader than that for pure helium. That makes it possible to determine the more precise position of the center of the peak. It appears that the shift of peak with respect to bulk helium is $\sim 0.2 \mathrm{mK}$, but at the same time the reproducibility of the measurements of the sound attenuation maximum in liquid helium from run to run is about $\sim 0.1 \mathrm{mK}$. Therefore based on these experiments we can say that even if the shift exists, it is less than or on the order of $0.2 \mathrm{mK}$. Note that Fig. 10 reflects the fact that the attenuation peak in bulk helium is $\sim 0.8 \mathrm{mK}$ below the $\lambda$-point [30].

\section{Discussion}

\subsection{Velocity of sound in Im-He solids}

Before performing this series of experiments, it was impossible to predict the characteristics of sound propagation in $\mathrm{Im}-\mathrm{He}$ samples created by introducing impurities into superfluid helium. According to the existing model these Im-He solids represent metastable phases formed by coalescing $\mathrm{Im}-\mathrm{He}$ clusters, in which helium is solidified as a result of large attractive van der Waals interactions between helium atoms and a central impurity particle. Therefore under the conditions of compact packing of these clusters one would expect an increase in elastic modulus for this material and also an increase of the speed of sound with respect to pure bulk liquid helium. A discovery of this effect was one of the first goals of this experiment. However we were not able to produce samples for which we could detect an increase in the speed of sound although we used our standard methods for creation of Im-He solids [6,7]. On the contrary, we observed a smaller speed of sound than in superfliud helium. The only exception were $\mathrm{D}_{2}-\mathrm{He}$ samples for which the sound velocity was the same as in helium to great precision. The observed decrease in the speed of sound can be explained by the creation of the porous structure of solid helium around the impurity particles. This is also supported by the fact that the behavior of the speed of sound resembles the fast sound mode in porous aerogel [28]. This mode is intermediate between first and fourth sound. Here the normal component is locked in a very compliant solid matrix so that the liquid and the aerogel fibers move together under mechanical and thermal gradients. McKeena et al. [28] developed a 
theory explaining the behavior of sound modes in aerogel, taking into account coupling between the normal component and the aerogel and its elasticity. The same features are observed in $\mathrm{Im}-\mathrm{He}$ samples.

\subsection{Attenuation of sound in $\mathrm{Im}-\mathrm{He}$ solids}

As we pointed out before, the behavior of the attenuation of sound in different heavy $\mathrm{Im}-\mathrm{He}$ solids has the same characteristic features. We observe the transition from the plateau with a small attenuation to the plateau with a bigger one, which ends with a maximum at the $\lambda$-point. This can be explained by the structure of this porous material, which is characterized by a wide distribution of pore sizes. Among these pores there are large channels in which the behavior of the helium is close to bulk helium. The existence of these pores is realistic especially if we take into account the method of collecting Im-He solids. This is a highly nonequilibrium process in which the impurity particles cooled by the helium vapor enter the superfluid helium where they stick together after random collisions with each other. So in this process, as the model of aggregation of the small particles into clusters predicts [31], highly ramified fractal structures are created.

The accumulation of $\mathrm{Im}-\mathrm{He}$ sample is characterized by the existence of a convective flow of helium which moves parts of the created condensate from a location where impurity particles first hit the surface of the helium to the bottom and to the walls of the cell. Later these small pieces of porous material stick together to form the Im-He solid. They do not coalesce homogeneously, however. Therefore macroscopic voids might be created between them, which can lead to the formation of large channels in the final condensate. We should notice that this Im-He solid preserves its form unless removed from the helium. Then it compresses by $60 \%$ [9].

Qualitatively the behavior of attenuation of sound in samples with this structure can be explained as follows: At the lowest temperatures the normal component of helium is locked to the solid matrix and the attenuation is small. Warming leads to a decrease of the viscous penetration depth $\delta_{\text {visc }}$ so that, when it is comparable to the pore size $R$ of our solid, the decoupling of a portion of the normal fluid occurs. Therefore sound attenuation caused by the friction of the layers of normal fluid as they become unlocked from the solid matrix starts rapidly increasing. Let us call the temperature, where this occurs, $T_{1}$. Continuous warmup leads to de- coupling of helium in a greater number of pores. We define $T_{2}$, as the temperature, where the sound propagates only in the large channels. There helium is almost like bulk helium and does not feel the effect of the walls. In addition sound can propagate through the smallest pores formed at the earliest stage of the creation of the sample. Helium in these pores is still locked to the solid matrix and attenuation is low and almost independent of temperature. This resembles the behavior of attenuation in bulk superfluid helium.

Calculating viscous penetration depths for $T_{1}$ and $T_{2}$ we can find the corresponding pore size $\left(\delta_{\text {visc }}=R\right)$. These results, inferred from Figs. 3,b and 4 , are shown in Table. Samples produced by injecting heavy impurities in superfluid helium are characterized by the presence of the pores of large size - from $150 \mathrm{~nm}$ to $820 \mathrm{~nm}$. On the other hand, the $\mathrm{D}_{2}-\mathrm{N}_{2}-\mathrm{He}$ sample has smaller pores $-100 \mathrm{~nm}$ to $140 \mathrm{~nm}$. It should be pointed out that from the analysis of the lowest temperature part of attenuation, we can estimate only the pores of larger size where a large attenuation is observed. Information about the smallest pores is much harder to obtain because after $T_{2}$, sound propagates not only through them but also through the large size channels.

Table

Values of $T_{1}$ and $T_{2}$ (see text) for different Im-He solids and corresponding pore sizes

\begin{tabular}{c|c|c|c|c}
\hline \hline Sample & $T_{1}, \mathrm{~K}$ & $R_{1}, \mathrm{~nm}$ & $T_{2}, \mathrm{~K}$ & $R_{2}, \mathrm{~nm}$ \\
\hline \hline $\mathrm{Kr}-\mathrm{He}$ & 1.20 & 530 & 1.56 & 210 \\
$\mathrm{Ne}-\mathrm{He}$ & 1.35 & 320 & 1.73 & 150 \\
$\mathrm{~N}_{2}-\mathrm{He}$ & 1.10 & 860 & 1.58 & 210 \\
$\mathrm{D}_{2}-\mathrm{N}_{2}-\mathrm{He}$ & 1.75 & 140 & 2.05 & 110 \\
$\mathrm{D}_{2}-\mathrm{He}$ & 2.05 & 110 & & \\
\hline \hline
\end{tabular}

This model is supported by investigation of sound attenuation in the same sample but at different frequencies. Figure 7 shows that a decrease in frequency leads to a decrease of attenuation. Also $T_{2}$ shifts to the higher temperature. For both frequencies $\delta_{\text {visc }}\left(T_{2}=1.48 \mathrm{~K}\right.$ at $\left.5 \mathrm{MHz}\right)$ is equal to $\delta_{\text {visc }}\left(T_{2}=1.6 \mathrm{~K}\right.$ at $\left.3 \mathrm{MHz}\right)$ which is in magnitude equal to $240 \mathrm{~nm}$.

Im-He samples formed in our experiments have a variety of different volume ratios between the porous part and the large channels. Compressing these samples leads to a decrease in the volume of the large channels, which in turn increases the 
attenuation, as was detected in the previous experiments [27]. The comparison of attenuation in freshly prepared samples and in those recycled above $T_{\lambda}$ showed that crossing the $\lambda$ transition always gives a somewhat larger attenuation caused by the compactification of Im-He solids. A much larger effect was observed in the $\mathrm{D}_{2}-\mathrm{N}_{2}-$ He sample during warmup to $3.4-3.5 \mathrm{~K}$ where a sudden drop in attenuation was observed. In addition it was seen visually that the sample volume changed from $1.7 \mathrm{~cm}^{3}$ to $0.14 \mathrm{~cm}^{3}$. We did not observe a similar effect in $\mathrm{N}_{2}-\mathrm{He}$ samples. Hence this change of attenuation is ascribed to the collapse of the porous structure formed by deuterium molecules. It seems as if a similar effect was detected in the work of Gordon et al. [32], where a significant decrease of concentration of deuterium atoms contained in a $\mathrm{D}_{2}-\mathrm{Ne}-\mathrm{He}$ sample was registered during the warmup from $1.8 \mathrm{~K}$ to $4.2 \mathrm{~K}$.

Analysis of sound propagation in Im-He solids shows that a distribution of the channels in superfluid helium is present, including very large channels, in which the helium behavior is close to that of bulk helium. Although the speed and attenuation of sound did not reveal any substantial suppression of $T_{c}$ in $\mathrm{N}_{2}-\mathrm{He}$ samples, we discovered a very small shift in $\mathrm{D}_{2}-\mathrm{N}_{2}-$ He samples, on the order of $0.2 \mathrm{mK}$ which is similar to that in a very light aerogel. In addition, a small broadening of the attenuation peak was detected for $\mathrm{N}_{2}-\mathrm{He}$ solid near the $\lambda$-point. The width of these peaks gives us information about the smallest pores in the samples. Using Josephson's relation [33] for helium in the channels of Im-He solids, namely

$$
\xi(t)=\xi_{0}|t|^{-\zeta}=\frac{k_{B} T_{c} m^{2}}{\hbar^{2} \rho_{s}(t)}
$$

we can find the correlation length at the temperature where the broadening of the attenuation peak starts. At this temperature $T$, the superfluidity in the pores (where the pore radius $R=\xi(T)$ ) starts breaking up. In equation (7) $m, k_{B}$ and $\hbar$ are the mass of a helium atom, Boltzmann's constant, and Planck's constant, respectively. From Fig. 7 we can say that the onset of broadening of the attenuation peak is at $T \sim 2.1 \mathrm{~K}$, which gives the characteristic size of the pores from the argument above as $R \sim 8 \mathrm{~nm}$. This is reasonably close to $(6 \pm 2) \mathrm{nm}$, the size of the clusters from which our Im- He solids are built [15]

Under certain favorable circumstances we were able to produce samples without any of the large channels and in this case we did not observe the

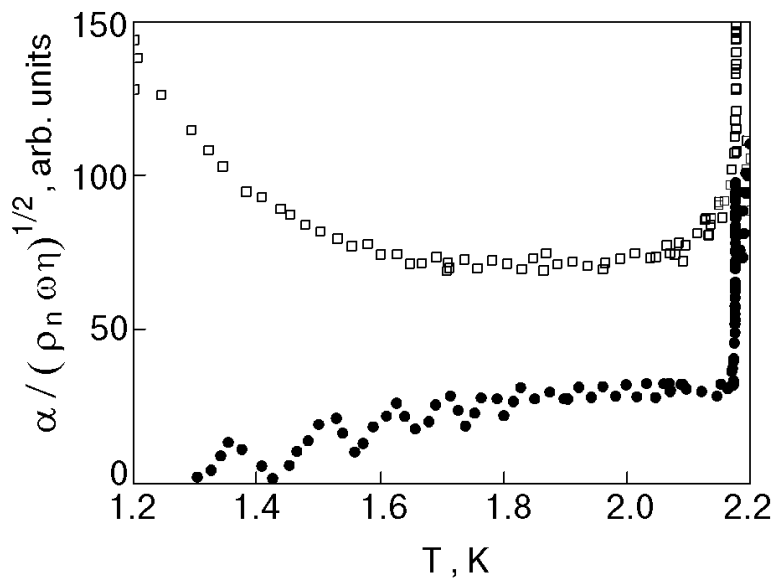

Fig. 11. Temperature dependence of ultrasound attenuation for $5.43 \mathrm{MHz}(\square)$ and $3.16 \mathrm{MHz}(\bullet)$

plateau in the temperature dependence of the attenuation (see Fig. 8). This idea was checked by plotting attenuation divided by $\left(\rho_{n} \omega \eta\right)^{1 / 2}$ as shown in Fig. 11. The constant straight line above $T \sim 1.6 \mathrm{~K}$ describes the high frequency behavior of helium in this particular porous sample, which implies that the energy loss is occurring on the entire surface in a thin layer of thickness $\delta_{\text {visc }}$. At a lower frequency this behavior starts at a higher temperature because the viscous penetration length is larger. If we compare the temperatures at which the graph in Fig. 11 levels off to a straight line, we get the same value of $\delta_{\mathrm{visc}} \sim 180 \mathrm{~nm}$ for 3 and $5 \mathrm{MHz}$ ultrasound.

\section{Summary}

Im-He solids have opened up a variety of intriguing possibilities for experimental investigations of the quantum properties of helium, as well as for studying atoms, molecules and small clusters stabilized in solidified helium. These studies provide a new perspective for matrix isolation in solidified helium. The big advantage of the Im-He solids is that a large variety of atoms or molecules can be used to build the «backbone» of the Im-He samples. It also appears that, depending on the preparation conditions, samples with different nanostructures can be prepared. To understand the properties of the $\mathrm{Im}-\mathrm{He}$ samples it is necessary to determine their microscopic structure.

In this work investigations of the velocity and attenuation of ultrasound were used to study the characteristics of Im-He samples formed by introducing different impurities in the volume of superfluid helium. For helium in Im-He samples the speed of sound is a little smaller than in bulk helium, and its temperature dependence is close to the fast sound mode in light aerogel [28]. The 
character of attenuation of ultrasound in helium in $\mathrm{Im}-\mathrm{He}$ samples is different from that in other porous materials like Vycor, porous gold, aerogel. The temperature dependence of ultrasound attenuation in $\mathrm{D}_{2}-\mathrm{He}$ samples is close to that in bulk helium whereas it is considerably different from the attenuation in heavy Im-He samples. This allows us to grow mixed solids, such as $\mathrm{D}_{2}-\mathrm{N}_{2}-\mathrm{He}$, in which the attenuation can be regulated by the content of the gasous mixture. For these mixed samples we produced and investigated the most «perfect» porous solids, which do not contain large channels of bulk helium.

From the analysis of attenuation of ultrasound in Im-He samples, we conclude that they have a wide distribution of pore sizes between $8 \mathrm{~nm}$ and $800 \mathrm{~nm}$, as well as large channels in which the behavior of helium is close to that of bulk liquid helium.

We investigated the behavior of the velocity and attenuation of ultrasound in Im-He samples near the $\lambda$-point where a broadening of the $\lambda$-peak is observed. The broadening increases with increasing sample density. A small shift of the transition temperature $(\sim 0.2 \mathrm{mK})$ was observed for $\mathrm{D}_{2}-\mathrm{N}_{2}-\mathrm{He}$ samples.

For a better understanding of the microscopic structure of Im-He samples, a study of low angle $\mathrm{x}$-ray scattering might be very helpful. The similarity between the behavior of ultrasound in $\mathrm{Im}-\mathrm{He}$ samples and in aerogel suggests the possibility of a fractal structure for Im-He solids. For studies of the critical behavior of helium in Im-He samples, investigations of second sound (low frequency sound) are the most appealing. We believe that this will allow the observation of the slow sound mode similar to the one observed in aerogel [28] and also the determination of the critical exponent for different Im-He solids.

\section{Acknowledgements}

We would like to thank NASA for its support through grant NAG 8-1445. We also wish to thank Drew Geller, John Beamish, Jeevak Parpia and John Reppy for very useful suggestions and discussions.

1. B. Tabbert, H. Gunter, and G. zu Putlits, J. Low Temp. Phys. 109, 653 (1997).

2. J. P. Toennies, and A. F. Vilesov, Annu. Rev. Phys. Chem. 49, 1 (1998)

3. S. I. Kanorsky and A. Weis, Adv. At. Mol. Opt. Phys. 38, 87 (1998).

4. E. B. Gordon and A. F. Shestakov, Fiz. Nizk. Temp. 26, 5 (2000) [Low Temp. Phys. 26, 1 (2000)].
5. E. B. Gordon, A. A. Pelmenev, O. F. Pugachev, and V. V. Khmelenko, JETP Lett. 37, 282 (1983).

6. E. B. Gordon, V. V. Khmelenko, E. A. Popov, A. A Pelmenev, and O. F. Pugachev, Chem. Phys. Lett. 155 , 301 (1989)

7. E. B. Gordon, V. V. Khmelenko, A. A. Pelmenev, E. A Popov, O. F. Pugachev, and A. F. Shestakov, Chem. Phys. 170, 411 (1993).

8. B. Palaszewski, L. S. Ianovski, and Patrick Carrik, $J$. Propulsion Power 14, 641 (1998).

9. R. E. Boltnev, E. B. Gordon, I. N. Krushinskaya, A. A Pelmenev, E. A. Popov, O. F. Pugachev, and V. V. Khmelenko, Fiz. Nizk. Temp. 18, 819 (1992) [Sov. J. Low Temp. Phys. 18, 576 (1992)].

10. R. E. Boltnev, E. B. Gordon, V. V. Khmelenko, I. N. Krushinskaya, M. V. Martynenko, A. A. Pelmenev, E. A. Popov, and A. F. Shestakov, Chem. Phys. 189, 367 (1994).

11. R. E. Boltnev, E. B. Gordon, I. N. Krushinskaya, M. V. Martynenko, A. A. Pelmenev, E. A. Popov, V. V. Khmelenko, and A. F. Shestakov, Fiz. Nizk. Temp. 23, 753 (1997) [Low Temp. Phys. 23, 567 (1997)].

12. R. E. Boltnev, I. N. Krushinskaya, A. A. Pelmenev, D. Yu. Stolyarov, and V. V. Khmelenko, Chem. Phys. Lett. 305, 217 (1999)

13. R. E. Boltnev, E. B. Gordon, V. V. Khmelenko, M. V. Martynenko, A. A. Pelmenev, E. A. Popov, and A. F. Shestakov, J. Chim. Phys. (France) 92, 362 (1995).

14. L. P. Mezhov-Deglin and A. M. Kokotin, JETP Lett. 70, 11 (1999).

15. V. Kiryukhin, B. Keimer, R. E. Boltnev, V. V. Khmelenko, and E. B. Gordon, Phys. Rev. Lett. 79, 1774 (1997).

16. J. D. Reppy, J. Low Temp. Phys. 87, 205 (1992).

17. J. M. Kosterlitz and D. J. Thouless, J. Phys. C6, 1131 (1973).

18. L. S. Goldner, N. Mulders, and G. Ahlers, J. Low Temp. Phys. 93, 131 (1993).

19. M. H. W. Chan, K. I. Blum, S. Q. Murphy, G. K. S. Wong, and J. D. Reppy, Phys. Rev. Lett. 61, 1950 (1988).

20. J. Yoon and M. H. W. Chan, Phys. Rev. Lett. 78, 4801 (1997)

21. J. Yoon, D. Sergatskov, J. Ma, N. Mulders, and M. H. W. Chan, Phys. Rev. Lett. 80, 1461 (1998).

22. K. L. Warner and J. R. Beamish, Phys. Rev. B36, 5698 (1987).

23. N. Mulders and J. R. Beamish, Phys. Rev. Lett. 62, 438 (1989).

24. K. Warner and J. R. Beamish, Phys. Rev. B50, 15896 (1994).

25. M. A. Biot, J. Acoust. Soc. Am. 28, 168 (1956).

26. M. A. Biot, J. Acoust. Soc. Am. 28, 179 (1956).

27. S. I. Kiselev, V. V. Khmelenko, D. A. Geller et al. J. Low Temp. Phys. 119, 357 (2000).

28. M. J. McKenna, T. Slawecki, and J. D. Maynard, Phys. Rev. Lett. 66, 1878 (1991).

29. E. B. Gordon, L. P. Mezhov-Deglin, O. F. Pugachev, and V. V. Khmelenko, Cryogenics 9, 555 (1976).

30. C. E. Chase, Phys. Fluids 1, 3 (1958).

31. P. Meakin, Ann. Rev. Phys. Chem. 39, 237 (1988).

32. E. B. Gordon, A. A. Pelmenev, O. F. Pugachev, and V. V. Khmelenko, Fiz. Nizk. Temp. 11, 563 (1985) [Sov. J. Low Temp. Phys. 11, 307 (1985)].

33. B. D. Josephson, Phys. Lett. 21, 608 (1966). 